\title{
Changes in airway inflammation during pulmonary exacerbations in patients with cystic fibrosis and primary ciliary dyskinesia
}

\author{
Felix Ratjen 1,2,3, Valerie Waters ${ }^{3,4}$, Michelle Klingel ${ }^{3}$, Nancy McDonald ${ }^{1}$, \\ Sharon Dell ${ }^{1,2,5}$, Timothy Ronan Leahy ${ }^{4}$, Yvonne Yau $^{2,6}$ and \\ Hartmut Grasemann ${ }^{1,2,3}$
}

\begin{abstract}
Affiliations: 'Division of Respiratory Medicine, Department of Paediatrics, Hospital for Sick Children, Toronto, Canada. ${ }^{2}$ University of Toronto, Toronto, Canada. ${ }^{3}$ Physiology and Experimental Medicine, Research Institute, Hospital for Sick Children, Toronto, Canada. ${ }^{4}$ Division of Infectious Diseases, Research Institute, Hospital for Sick Children, Toronto, Canada. ${ }^{5}$ Child Health Evaluative Sciences, Research Institute, Hospital for Sick Children, Toronto, Canada. ${ }^{6}$ Division of Microbiology, Dept of Pediatric Laboratory Medicine, Hospital for Sick Children, Toronto, Canada.
\end{abstract}

Correspondence: Felix Ratjen, Division of Respiratory Medicine, Department of Paediatrics, The Hospital for Sick Children, 555 University Avenue, Toronto Ontario M5G 1X8, Canada. E-mail: felix.ratjendasickkids.ca

ABSTRACT Lung disease in patients with both primary ciliary dyskinesia (PCD) or cystic fibrosis (CF) is associated with impaired mucociliary clearance; however, clinical outcomes are typically worse in $\mathrm{CF}$ patients. We assessed whether CF and PCD patients differ in inflammatory response in the airways during pulmonary exacerbation.

We first studied clinically stable PCD patients with a spectrum of bacterial pathogens to assess inflammatory response to different pathogens. Subsequently, PCD and CF patients with similar bacterial pathogens were studied at the time of a pulmonary exacerbation and after 21 days of antibiotics treatment. Qualitative and quantitative microbiology, cell counts, interleukin-8 concentrations, and neutrophil elastase activity were assessed in sputum samples obtained before and after treatment.

In stable PCD patients, no significant differences were found in sputum inflammatory markers between individuals colonised with different bacterial pathogens. Pulmonary exacerbation severity assessed by a pulmonary exacerbation score and lung function decline from their previous baseline did not differ between CF and PCD patients. Bacterial density for Staphylococcus aureus and Haemophilus influenzae was higher in CF versus PCD $(\mathrm{p}<0.05)$, but absolute neutrophil counts were higher in PCD patients $(p=0.02)$. While sputum elastase activity was similar in PCD and CF at the time of exacerbation, it decreased with antibiotic therapy in PCD $(p<0.05)$ but not CF patients.

PCD patients differ from those with CF in their responses to treatment of pulmonary exacerbations, with higher neutrophil elastase activity persisting in the CF airways at the end of treatment.

@ERSpublications

PCD patients differ from those with $\mathrm{CF}$ in their responses to treatment of pulmonary exacerbations http://ow.ly/TtM54

Received: Aug 212015 | Accepted after revision: Oct 052015 | First published online: Nov 192015

Support statement: This study was supported by CIHR (GPG-102171). Funding information for this article has been deposited with FundRef.

Clinical trial: This study is registered at www.clinicaltrials.gov with identifier number NCT01155115.

Conflict of interest: None declared.

Copyright OERS 2016 


\section{Introduction}

Lung disease in people suffering from cystic fibrosis (CF) or primary ciliary dyskinesia (PCD) is characterised by impaired mucociliary clearance and recurrent, persistent bacterial infections contributing to the development of bronchiectasis [1,2]. Despite similarities in pathophysiology, outcomes differ between the two diseases, with the course of lung disease being more favourable for PCD patients [3-6]. A number of factors have been proposed to account for this difference, including better cough clearance in PCD, as the ciliary abnormalities in PCD do not result in the same degree of airway surface liquid depletion seen in CF [1,2]. In addition, differences in the age of onset of Pseudomonas aeruginosa infection have been described, but it is currently unclear whether this is the cause or the result of less severe disease manifestations [3].

Both CF and PCD are associated with a neutrophil-dominated inflammation of the airways [7, 8]. The evolution of airway inflammation over time, the effect of acute exacerbations as well as the link between inflammation and subsequent course of lung disease have been extensively studied in CF [8-12]. However, similar information is lacking for PCD patients. Cross-sectional studies suggest that while both diseases are associated with a similar degree of airway neutrophilia, interleukin (IL)-8 sputum concentrations are higher in PCD than CF patients [7]. The same study suggested there was lower neutrophil elastase (NE) activity in PCD compared with CF patients, which is of note as NE activity in sputum has been shown to be associated with lung function decline over time [10] and NE activity in bronchoalveolar lavage (BAL) fluid predicts the subsequent development of bronchiectasis in CF [12]. Although the response to inflammatory stimuli in CF is exaggerated and resolution of inflammation impaired, especially during pulmonary exacerbations [13-15], it is currently unclear whether this inflammatory profile as well as response to treatment is similar in PCD. The aims of this study were therefore to assess airway inflammation in clinically stable PCD patients, and to compare measures of airway inflammation in CF and PCD patients at the time of and after treatment for a pulmonary exacerbation.

\section{Methods}

Study participants

The study was approved by the Research Ethics Board at the Hospital for Sick Children (REB number 1000013966) and was registered with www.clinicaltrials.gov (NCT01155115). Study participants included patients with PCD and CF who were followed in subspecialty clinics at the Hospital for Sick Children, Toronto, Canada. We first studied a cohort of PCD patients at routine outpatient visits at the time of clinical stability. Patients were eligible if they met the following inclusion criteria: 1) diagnosis of PCD as defined by clinical signs and symptoms consistent with the disease, a low nasal nitric oxide as well as evidence for structural ciliary defects on electron microscopy of a nasal or bronchial brush sample and/or two known disease-causing mutations on genetic testing; 2) informed consent and verbal assent (as appropriate) provided by the subject's parent or legal guardian and the subject; 3 ) at least 6 years of age at enrolment; 4) able to perform reproducible spirometry meeting American Thoracic Society (ATS) standards; 5) ability to produce sputum spontaneously; 6) clinically stable at the time of assessment. Exclusion criteria for study entry were: 1) use of intravenous antibiotics or oral quinolones within previous 14 days; 2) use of inhaled antibiotics within the previous 28 days; 3 ) recent history of pneumothorax or haemoptysis.

In addition, a subset of PCD patients as well as patients with CF were studied at the time of a pulmonary exacerbation (defined as an increase in respiratory symptoms treated with oral antibiotics) and at the end of a 3-week course of antibiotic therapy. CF patients had to have clinical features of CF and a documented sweat chloride $>60 \mathrm{mEq} \cdot \mathrm{L}^{-1}$ by quantitative pilocarpine iontophoresis test or genetic testing showing two well-characterised disease-causing mutations. Similar to the PCD population, patients had to be at least 6 years of age at enrolment, able to perform reproducible spirometry meeting ATS standards as well as able to produce sputum spontaneously. Patients receiving intravenous antibiotics, inhaled or oral antibiotics, or patients with a recent history of pneumothorax or haemoptysis were excluded from the study. To allow for comparisons between the groups, only patients with a past history of positive airway cultures for Staphylococcus aureus or Haemophilus influenzae, which are common pathogens in both diseases, were included; patients with P. aeruginosa or Burkholderia cepacia complex infection were excluded from this part of the study as these are relatively uncommon in paediatric PCD patients. At the time of pulmonary exacerbation, patients were assessed clinically and performed spirometry according to ATS criteria. No established pulmonary exacerbation scoring system exists for patients with PCD; we therefore used the CF Akron pulmonary exacerbation score as a measure of exacerbation severity in both patients groups [16].

Spontaneously expectorated sputum was obtained for microbiological tests and analysis of cytokine levels at study visits. Bacterial culture and susceptibility testing were performed at the beginning of a pulmonary exacerbation, as per standard of care. Standardised operating procedures developed by the North American CF Therapeutics Development Network were followed for the test technique and sputum processing. 
Important details include the use of standard infection control procedures for the handling of respiratory secretions, adequate supervision and coaching of the child to expectorate a specimen, and immediate transport (within $1 \mathrm{~h}$ ) of the sputum to the laboratory for processing [17]. Sputum IL-8 concentrations were measured by ELISA (R\&D Systems, Minneapolis, MN, USA) and NE activity similar to that reported previously [18].

Patients with CF and PCD with pulmonary exacerbations were treated with a course of oral antibiotics for 3 weeks as prescribed by their primary care physician. Patients returned for a follow-up visit 4 weeks after the onset of the pulmonary exacerbation, at which time point sputum and pulmonary function measurements were repeated.

\section{Statistical analysis}

Descriptive statistics were used to summarise the cross-sectional data of the PCD population at the outpatient visit when clinically stable. Comparisons between CF and PCD patients were performed using a Kruskal-Wallis test to compare median values of continuous data; Chi-squared statistics were used to compare proportions between groups. Treatment effects were assessed by one-way ANOVA where appropriate. Correlations between variables were evaluated using the Pearson correlation coefficient. Statistical analyses were performed using Stata 13.1 (StataCorp, College Station, TX, USA).

\section{Results}

Sputum measures of airway inflammation were obtained from 35 PCD patients with a median age (range) of 13.7 (5.8-17.9) years at a routine clinic visit while clinically stable; the characteristics of the population are shown in table 1. Lung function ranged from moderate airway obstruction to normal with a median (range) forced expiratory volume in $1 \mathrm{~s}(\mathrm{FEV})$ of 83 (53-132) \% pred. H. influenzae $(\mathrm{n}=14)$ and S. aureus (meticillin-sensitive) $(\mathrm{n}=10)$ were the predominant organisms in sputum cultures; five patients were positive for $P$. aeruginosa and 10 only grew normal flora. Sputum analysis demonstrated neutrophilic airway inflammation with a median neutrophil percentage of $96 \%$. Median (range) IL-8 concentration was $181.4(4.3-990.9) \mathrm{ng} \cdot \mathrm{mL}^{-1}$ and NE activity $12.4(0.3-308.7) \mathrm{nmol} \cdot \mathrm{min}^{-1}$. No correlation was observed between sputum markers of airway inflammation and measures of lung function in this cross-sectional data set (data not shown). In patients with positive airway cultures for either S. aureus or H. influenzae, there were no significant differences in sputum total cell counts, percentage neutrophils, IL-8 concentrations or NE activity between the two groups (table 2).

Recruitment of patients studied before and after exacerbations was targeted on patients with previous culture positivity for either $H$. influenzae and/or S. aureus. $35 \mathrm{CF}$ patients were approached and all agreed to participate in the study. 18 patients dropped out either due to inability to produce sputum at follow-up

\section{TABLE 1 Characteristics of stable primary ciliary dyskinesia patients}

\begin{tabular}{|c|c|c|}
\hline Measure & $\mathrm{n}$ & \\
\hline \multicolumn{3}{|l|}{ Demographics } \\
\hline Age years & 35 & $13.7(5.8-17.9)$ \\
\hline Male:female $n$ & 35 & $20: 15$ \\
\hline \multicolumn{3}{|l|}{ Inflammatory markers } \\
\hline $\mathrm{IL}-8 \mathrm{ng} \cdot \mathrm{mL}^{-1}$ & 35 & $181.4(4.27-990.9)$ \\
\hline $\mathrm{NE}$ activity $\mathrm{nmol} \cdot \mathrm{min}^{-1}$ & 35 & $12.4(0.25-308.7)$ \\
\hline Total cell count $\times 10^{6}$ & 35 & $1.6(0.02-34.2)$ \\
\hline Neutrophils \% & 16 & $95.5(65-99.8)$ \\
\hline \multicolumn{3}{|l|}{ Pulmonary function ${ }^{\#}$} \\
\hline $\mathrm{FEV}_{1} \%$ predicted & 33 & $83(53-132)$ \\
\hline FVC \% predicted & 33 & $94(58-119)$ \\
\hline FEF25-75\% \% predicted & 33 & $66(24-170)$ \\
\hline \multicolumn{3}{|l|}{ Microbiology\# } \\
\hline Haemophilus influenzae & 35 & $14(40)$ \\
\hline Staphylococcus aureus & 35 & $10(29)$ \\
\hline Pseudomonas aeruginosa & 35 & $5(14)$ \\
\hline Streptococcus spp. & 35 & $2(6)$ \\
\hline Usual flora & & $10(29)$ \\
\hline
\end{tabular}


TABLE 2 Sputum inflammatory markers according to bacterial pathogens ${ }^{\#}$

\begin{tabular}{lccc} 
& Haemophilus influenzae & Staphylococcus aureus & Usual flora \\
\hline Subjects n & 9 & 7 & 10 \\
Age years & $11.9(5.8-16.0)$ & $15.8(10.2-17.9)$ & $14.4(7.5-17.7)$ \\
FEV \% predicted & $85(59-99)$ & $84(74-113)$ & $90(58-132)$ \\
FVC \% predicted & $93(89-105)$ & $99(86-109)$ & $96(65-119)$ \\
FEF25-75\% \% predicted & $70(24-86)$ & $62(50-96)$ & $73(38-170)$ \\
Total cell count $\mathbf{\times 1 0 ^ { 6 }}$ & $1.5(0.1-25.1)$ & $2.1(0.3-4.2)$ & $1.2(0.3-10.3)$ \\
Neutrophils \% & $95.6(65.0-99.8)$ & $92.4(88-96.8)$ & $94.3(77.3-98.5)$ \\
IL-8 $\mathbf{n g} \cdot \mathbf{m L}^{-1}$ & $418.7(4.3-990.9)$ & $69.6(5.4-400.7)$ & $206.8(106.0-508.0)$ \\
NE nmol-min & & $10.1(0.3-30.3)$ & $4.7(1.4-113.9)$ \\
\hline
\end{tabular}

Data are presented as median (range), unless otherwise stated. ${ }^{\#}$ : only patients with single pathogens included. FEV1: forced expiratory volume in $1 \mathrm{~s}$; FVC: forced vital capacity; FEF25-75\%: forced expiratory flow at $25-75 \%$ of FVC; IL: interleukin; NE: neutrophil elastase.

$(n=6)$, growth of other pathogens at the time of exacerbation $(n=6)$, lack of growth of pathogens in sputum samples $(n=3)$, admission to hospital $(n=2)$ or withdrawal from the study $(n=1)$. 15 PCD patients agreed to participate in the study of which four patients grew no pathogens at the time of exacerbation with 11 patients remaining in the study. The characteristics of the study population are shown in table 3 .

Median FEV1 was lower in the PCD patients, but this difference was not statistically significant. Both groups had similar drops in FEV1 from their previous baseline; median absolute change from baseline in FEV 1 was $-18 \%$ in CF and $-22 \%$ in PCD. There were no differences in the pulmonary exacerbation score between both patient groups. Total cell counts in sputum were numerically higher in PCD ( $\mathrm{p}=0.06)$ and the percentage of neutrophils similar in both groups, but PCD patients had higher absolute neutrophil counts $(p=0.02)$. Sputum concentrations of IL- 8 were similar, while sputum total bacterial density was higher in CF versus PCD patients (table 3).

Clinical response to oral antibiotic therapy was similar in both patient groups, with pulmonary exacerbation scores improving by a median (range) of $-3(-5.7-1.2)$ in CF and $-1.5(-4.2-0)$ in PCD patients $(\mathrm{p}=0.29)$, and FEV1 increasing by $15.5(-3-38) \%$ in CF and 10 (1-17) \% in PCD patients $(\mathrm{p}=0.40)$. Bacterial density, which was higher at baseline in CF, decreased significantly in CF, but not in PCD (figure 1). Changes in sputum markers with treatment of pulmonary exacerbations are shown in figures $2-5$. Total cell counts as well as absolute neutrophil counts in sputum showed a trend toward improvement in PCD patients, but

TABLE 3 Patient characteristics at the time of pulmonary exacerbation

\begin{tabular}{|c|c|c|c|}
\hline & Cystic fibrosis & Primary ciliary dyskinesia & p-value \\
\hline Subjects $\mathbf{n}$ & 17 & 11 & \\
\hline Age years & $12.0(6.9-16.7)$ & $11.0(6.8-15.3)$ & 0.94 \\
\hline Male & $6(35)$ & $6(54)$ & 0.32 \\
\hline Body mass index centile & $56.5(4-90)$ & $46(0.2-91)$ & 0.75 \\
\hline Pancreatic insufficient & $11(65)$ & $0(0)$ & 0.001 \\
\hline \multicolumn{4}{|l|}{ Pulmonary function } \\
\hline FEV $1 \%$ predicted & $74(45-104)$ & $65(39-86)$ & 0.10 \\
\hline FVC \% predicted & $90(51-109)$ & $77(59-102)$ & 0.09 \\
\hline Change in FEV 1 from baseline \% & $-18(-36-6)$ & $-22(-50--4)$ & 0.66 \\
\hline Change in FVC from baseline $\%$ & $-14(-36-7)$ & $-15(-36-8)$ & 0.69 \\
\hline Pulmonary exacerbation score & $4.2(2.7-7.1)$ & $4.1(2.7-8.7)$ & 0.32 \\
\hline \multicolumn{4}{|l|}{ Inflammatory markers } \\
\hline Bacterial density $\log _{10} \mathrm{CFU} \cdot \mathrm{mL}^{-1}$ & $7.4(5.4-8.5)$ & $5.1(0.04-35.4)$ & 0.02 \\
\hline Total cell count $\times 10^{6}$ & $1.6(0.04-15.3)$ & $6.4(0.04-35.4)$ & 0.06 \\
\hline Neutrophils \% & $96.1(43.3-100)$ & $96.4(88.3-98.8)$ & 0.86 \\
\hline Neutrophils absolute $\times 10^{6}$ & $2.5(0.2-15.2)$ & $11.4(0.8-34.4)$ & 0.02 \\
\hline $\mathrm{IL}-8 \mathrm{ng} \cdot \mathrm{mL}^{-1}$ & $73.5(4.4-365.9)$ & $90.8(64.8-825.9)$ & 0.10 \\
\hline $\mathrm{NE}$ activity $\mathrm{nmol} \cdot \mathrm{min}^{-1}$ & $46.3(4.5-224.5)$ & $91.4(11.8-166.4)$ & 0.23 \\
\hline
\end{tabular}

Data are presented as median (range) or $\mathrm{n}(\%)$, unless otherwise stated. FEV1: forced expiratory volume in $1 \mathrm{~s}$; FVC: forced vital capacity; IL: interleukin; NE: neutrophil elastase. 


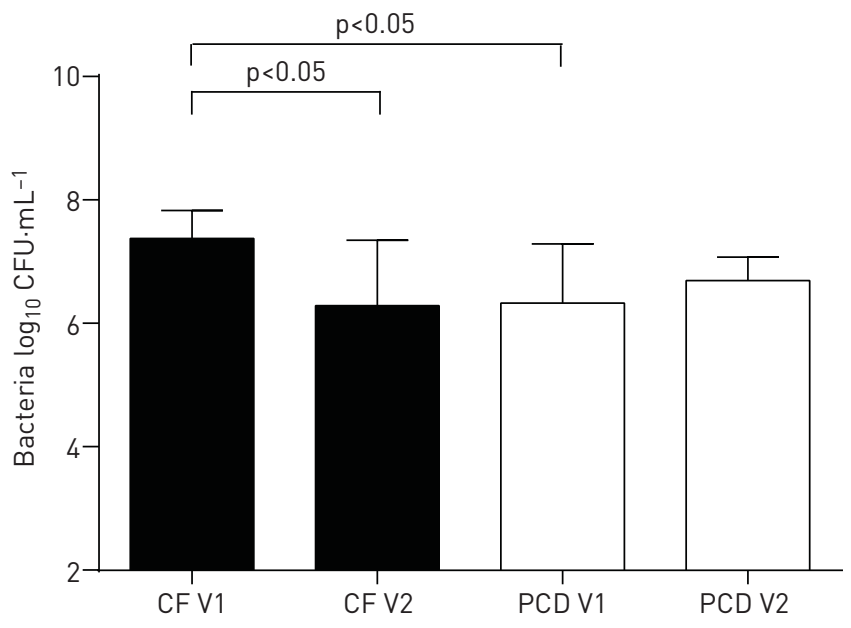

FIGURE 1 Bacterial density in sputum at the time of pulmonary exacerbation (V1) and after a 3-week course of treatment with oral antibiotics (V2) in cystic fibrosis (CF) patients and patients with primary ciliary dyskinesia (PCD). Data are presented as median and interquartile ranges. Bacterial density was higher in CF than PCD patients at the time of exacerbation and decreased significantly with treatment; no changes were observed in PCD patients.

remained unchanged in CF patients (figures 2 and 3). Sputum IL-8 concentrations did not change significantly with treatment in either group (figure 4). NE activity after antibiotic treatment, when compared with pre-treatment, had decreased in PCD; the change was not significant in CF patients (figure 5). There was a weak but not statistically significant correlation between improvements in FEV1 and decreases in sputum NE activity for CF and PCD patients combined $\left(\mathrm{r}^{2}=0.1657 ; \mathrm{p}=0.07\right)$ and $\mathrm{CF}$ patients alone $\left(\mathrm{r}^{2}=0.2185 ; \mathrm{p}=0.08\right)$.

\section{Discussion}

This is the first study to compare airway inflammation in CF and PCD patients in response to treatment of pulmonary exacerbations. We first demonstrated similar degrees of airway inflammation between clinically stable PCD patients infected with different bacterial pathogens. In patients assessed during pulmonary exacerbations, we found no significant differences in the clinical response to treatment between CF and PCD patients as assessed by lung function testing and pulmonary exacerbation score. At the time of exacerbation, bacterial density was higher in CF compared with PCD, whereas absolute neutrophil counts were higher in PCD patients. Treatment of pulmonary exacerbations with oral antibiotics resulted in a significant reduction in bacterial density in CF patients, while no significant changes were seen in PCD

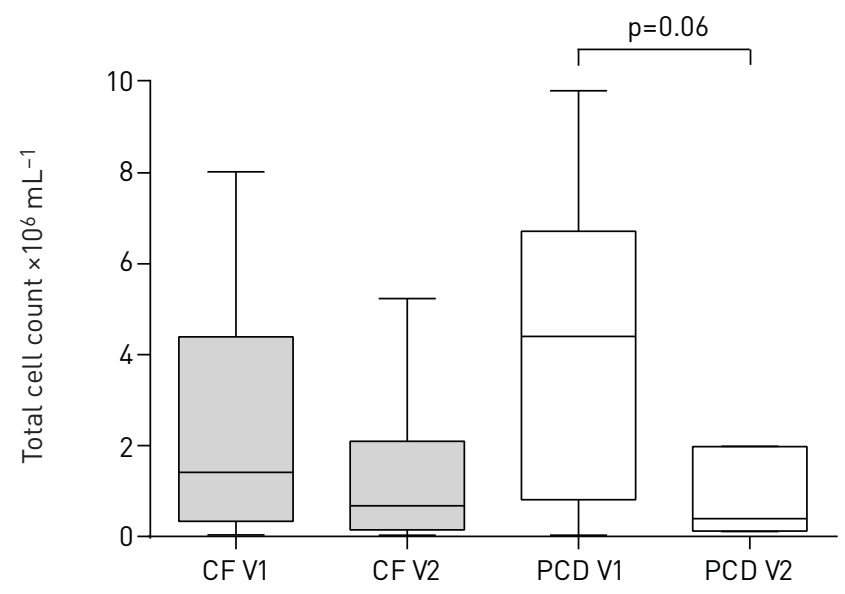

FIGURE 2 Total cell count in sputum at the time of pulmonary exacerbation (V1) and after a 3-week course of treatment with oral antibiotics (V2) in cystic fibrosis (CF) patients and patients with primary ciliary dyskinesia (PCD). Data are presented as box-and-whisker plots showing median and ranges. Total cell counts were similar in $\mathrm{CF}$ and $\mathrm{PCD}$ patients at the time of exacerbation, decreases were numerically greater, but nonsignificant in PCD patients $(p=0.06)$. 


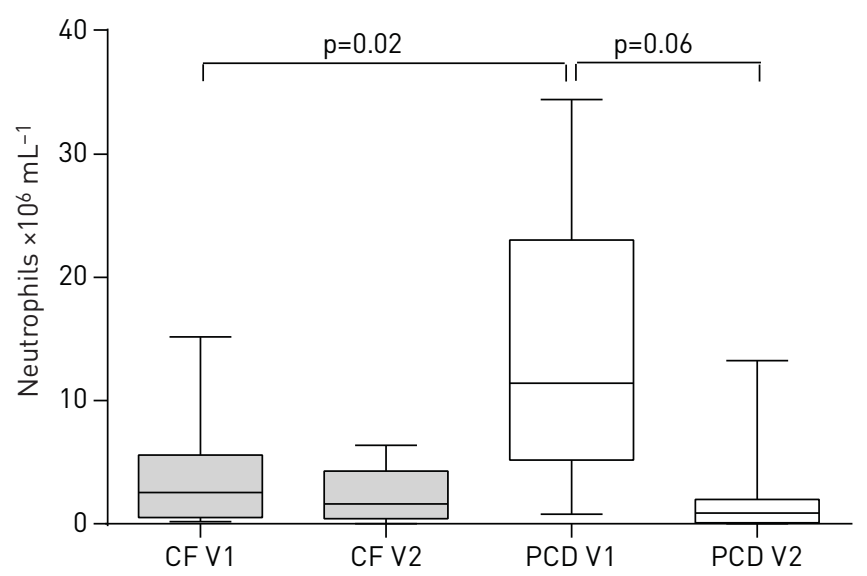

FIGURE 3 Absolute neutrophil count in sputum at the time of pulmonary exacerbation (V1) and after a 3-week course of treatment with oral antibiotics (V2) in cystic fibrosis (CF) patients and patients with primary ciliary dyskinesia (PCD). Data are presented as box-and-whisker plots showing median and ranges. Absolute neutrophil counts were higher in PCD than in CF patients at the time of exacerbation, decreases were numerically greater, but nonsignificant in PCD patients ( $p=0.06)$.

patients. Measures of airway inflammation did not change in CF patients, but sputum NE activity decreased significantly in PCD patients. These data indicate that differences in airway inflammation exist between CF and PCD patients at the time of pulmonary exacerbations and in response to antibiotic treatment.

Previous in vitro and in vivo studies have suggested an exaggerated inflammatory response of the CF airways [19-21]. In our study, absolute sputum neutrophil counts were higher in PCD than CF patients at the time of pulmonary exacerbation, despite similar clinical severity and similar decreases in lung function. Although not statistically significant, PCD was associated with higher sputum concentrations of IL-8, a major neutrophil attractant, which persisted at high levels at the end of treatment. This supports previous cross-sectional data which also found IL-8 to be higher in PCD versus CF patients [7]. Both epithelial cells and neutrophils are known sources of IL- 8 and the current study was unable to differentiate its cellular origin. The high levels of IL-8 and increased neutrophil counts in PCD patients at the time of pulmonary exacerbation compared with $\mathrm{CF}$ in conjunction with a high neutrophil load make it unlikely that the sustained inflammatory response in CF is unique to CF. While evidence has linked the exaggerated inflammatory response to CF transmembrane conductance regulator (CFTR) deficiency, it is not known whether other diseases such as PCD are associated with secondary CFTR deficiency or related ion channel abnormalities as previously described in smokers [22].

Sustained airway inflammation could reflect both an inadequate response to inflammatory triggers or a defect in the inhibition of inflammation, or both. Studies of human bronchial epithelial cells and animal studies have suggested a link between CFTR mutations and impaired resolution of inflammation [13-15]. Reduced responses in anti-inflammatory pathways involving cytokines such as IL-10 as well as resolvins

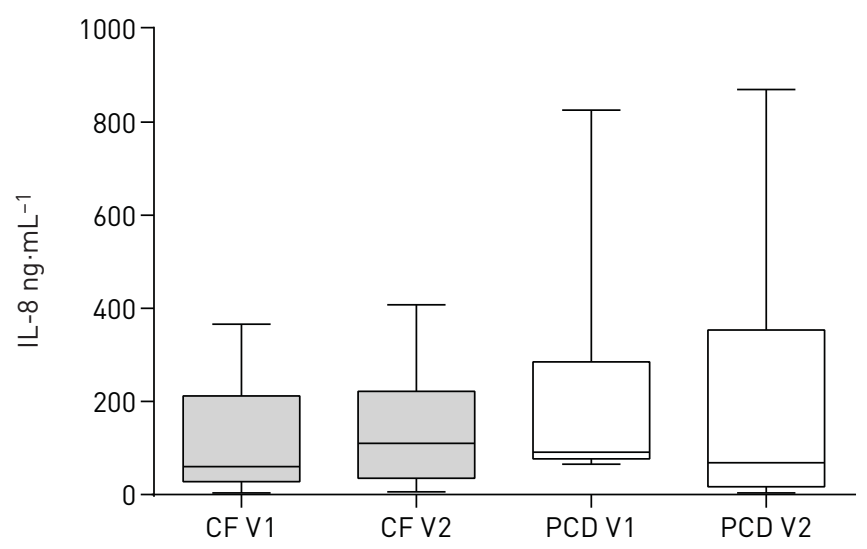

FIGURE 4 Interleukin (IL)-8 concentrations in sputum at the time of pulmonary exacerbation (V1) and after a 3 -week course of treatment with oral antibiotics (V2) in cystic fibrosis (CF) patients and patients with primary ciliary dyskinesia (PCD). Data are presented as box-and-whisker plots showing median and ranges. 


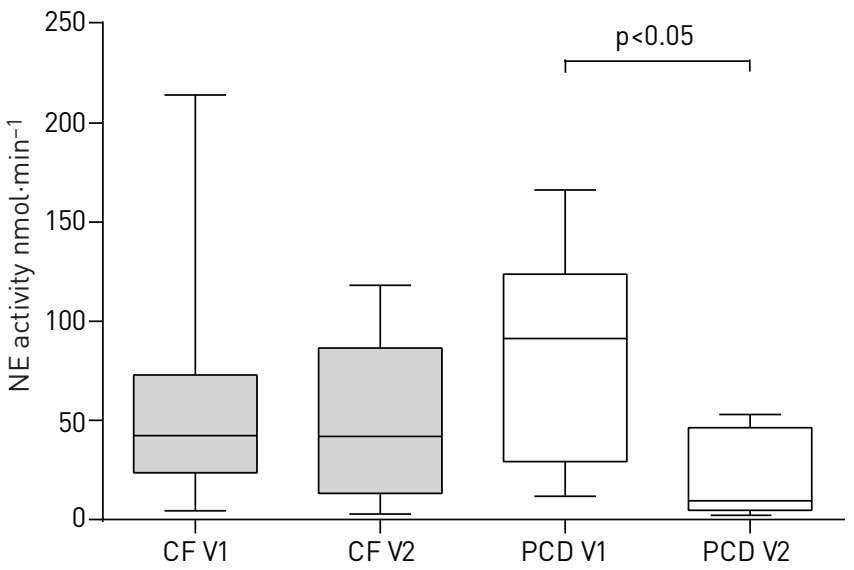

FIGURE 5 Neutrophil elastase (NE) activity in sputum at the time of pulmonary exacerbation (V1) and after a 3-week course of treatment with oral antibiotics (V2) in cystic fibrosis (CF) patients and patients with primary ciliary dyskinesia (PCD). Data are presented as box-and-whisker plots showing median and ranges. NE activity was similar in CF and PCD at the time of pulmonary exacerbation and decreased significantly in PCD, but not CF patients.

have been implicated as potential causes for this finding in CF [13, 22]. The current study would support this concept as NE activity, while significantly reduced with antibiotic treatment in PCD patients, did not change with antibiotic therapy in CF patients, despite reductions in bacterial density. This also raises the question whether oral antibiotic therapy in the context of pulmonary exacerbations in CF patients, although significantly reducing bacterial load, is sufficient to decrease airway inflammation.

There is mounting evidence that NE is a key element driving inflammation induced lung damage in $\mathrm{CF}$ [6]. Sputum NE activity is a predictor of future lung function decline [10]. In addition, sputum NE activity predicts failure of lung function recovery after treatment of a CF pulmonary exacerbation, which is consistent with the present study in which lung function response correlated with changes in NE activity [11]. In addition, higher $\mathrm{NE}$ activity is associated with a shorter time to subsequent pulmonary exacerbation [11]. BAL fluid NE activity is also an independent predictor for the development of bronchiectasis in infants with CF [12]. Therefore, persistence of high NE activity in CF airways after pulmonary exacerbations could potentially be an indicator for future lung function decline, thus providing a potential explanation for the reported steeper decline in lung function in CF versus PCD patients.

The overall changes in inflammatory markers observed in this study were smaller than previously reported for studies focussing on CF patients receiving intravenous antibiotics, which are usually reserved for more severe exacerbations and/or bacterial pathogens for which limited oral antibiotic options are available. These previous studies using intravenous antibiotic therapy in CF patients have shown more pronounced reductions in both bacterial load, as measured by CFUs, and markers of inflammation $[11,23,24]$. There are limited data comparing different routes of antibiotic therapy, but a small study in CF patients suggested that reduction of inflammation is greater with intravenous versus inhaled antibiotic therapy [25]. Similar data are lacking for oral antibiotic therapy, but it is tempting to speculate that oral antibiotic therapy may be less efficacious than intravenous therapy in addressing airway inflammation in patients with pulmonary exacerbations, but further evidence is needed to support this hypothesis.

The study has a number of important limitations. The overall number of patients was small, making it possible that the observed difference occurred by chance. Additionally, due to limited power, some true differences may appear insignificant and thus have been missed. The analysis was limited to patients with $S$. aureus and $H$. influenzae infections. The inflammatory responses may differ for other bacterial pathogens; however, we did not find any evidence for this in the cross-sectional analysis of stable PCD patients, which is consistent with previous CF studies [26-29]. Studies using molecular techniques have demonstrated a wide spectrum of bacteria in both CF and non-CF bronchiectasis that are not detected on routine cultures, and it is likely that these bacteria contribute to the inflammatory response. Adherence to antibiotic therapy was not tracked in the study and may potentially have confounded the results.

In summary, we observed higher neutrophil burden in PCD compared with CF patients at the time of a pulmonary exacerbation, but bacterial load was higher in CF patients. Antibiotic therapy reduced bacterial counts in CF, but not PCD, whereas NE activity decreased in PCD, but not in CF patients. Further evidence is needed to assess whether these differences in airway inflammation affect lung function decline 
and long-term outcome in CF compared with PCD patients, and whether the observed differences in response to oral treatment can be overcome by intravenous antibiotics.

\section{References}

Ratjen F, Bell SC, Rowe SM, et al. Cystic fibrosis. Nat Rev Dis Prim 2015; doi: 10.1038/nrdp.2015.10.

2 Knowles MR, Daniels LA, Davis SD, et al. Primary ciliary dyskinesia. Recent advances in diagnostics, genetics, and characterization of clinical disease. Am J Respir Crit Care Med 2013; 188: 913-922.

3 Noone PG, Leigh MW, Sannuti A, et al. Primary ciliary dyskinesia: diagnostic and phenotypic features. Am J Respir Crit Care Med 2004; 169: 459-467.

4 Marthin JK, Petersen N, Skovgaard LT, et al. Lung function in patients with primary ciliary dyskinesia: a cross-sectional and 3-decade longitudinal study. Am J Respir Crit Care Med 2010; 181: 1262-1268.

5 Magnin ML, Cros P, Beydon N, et al. Longitudinal lung function and structural changes in children with primary ciliary dyskinesia. Pediatr Pulmonol 2012; 47: 816-825.

6 Stanojevic S, Bilton D, McDonald A, et al. Global Lung Function Initiative equations improve interpretation of FEV1 decline among patients with cystic fibrosis. Eur Respir J 2015; 46: 262-264.

7 Bush A, Payne D, Pike S, et al. Mucus properties in children with primary ciliary dyskinesia: comparison with cystic fibrosis. Chest 2006; 129: 118-123.

8 Cantin AM, Hartl D, Konstan MW, et al. Inflammation in cystic fibrosis lung disease: pathogenesis and therapy. J Cyst Fibros 2015; 14: 419-430.

9 Grasemann H, Ratjen F. Early lung disease in cystic fibrosis. Lancet Respir Med 2013; 1: 148-157.

10 Sagel SD, Wagner BD, Anthony MM, et al. Sputum biomarkers of inflammation and lung function decline in children with cystic fibrosis. Am J Respir Crit Care Med 2012; 186: 857-865.

11 Waters VJ, Stanojevic S, Sonneveld N, et al. Factors associated with response to treatment of pulmonary exacerbations in cystic fibrosis patients. J Cyst Fibros 2015; 14: 755-762.

12 Sly PD, Gangell CL, Chen L, et al. Risk factors for bronchiectasis in children with cystic fibrosis. N Engl J Med 2013; 368: 1963-1970.

13 Bonfield TL, Konstan MW, Burfeind P, et al. Normal bronchial epithelial cells constitutively produce the anti-inflammatory cytokine interleukin-10, which is downregulated in cystic fibrosis. Am J Respir Cell Mol Biol 1995; 13: 257-261.

14 Bensalem N, Ventura AP, Vallée B, et al. Down-regulation of the anti-inflammatory protein annexin A1 in cystic fibrosis knock-out mice and patients. Mol Cell Proteomics 2005; 4: 1591-1601.

15 Luciani A, Villella VR, Esposito S, et al. Defective CFTR induces aggresome formation and lung inflammation in cystic fibrosis through ROS-mediated autophagy inhibition. Nat Cell Biol 2010; 12: 863-875.

16 Kraynack NC, Gothard MD, Falletta LM, et al. Approach to treating cystic fibrosis pulmonary exacerbations varies widely across US CF care centers. Pediatr Pulmonol 2011; 46: 870-881.

17 Sagel SD, Kapsner R, Osberg I, et al. Airway inflammation in children with cystic fibrosis and healthy children assessed by sputum induction. Am J Respir Crit Care Med 2001; 164: 1425-1431.

18 Bieth J, Spiess B, Wermuth CG. The synthesis and analytical use of a highly sensitive and convenient substrate of elastase. Biochem Med 1974; 11: 350-357.

19 Heeckeren A, Walenga R, Konstan MW, et al. Excessive inflammatory response of cystic fibrosis mice to bronchopulmonary infection with Pseudomonas aeruginosa. J Clin Invest 1997; 100: 2810-2815.

20 Kube D, Sontich U, Fletcher D, et al. Proinflammatory cytokine responses to P. aeruginosa infection in human airway epithelial cell lines. Am J Physiol Lung Cell Mol Physiol 2001; 280: L493-L502.

21 Muhlebach MS, Stewart PW, Leigh MW, et al. Quantitation of inflammatory responses to bacteria in young cystic fibrosis and control patients. Am J Respir Crit Care Med 1999; 160: 186-191.

22 Cantin AM, Hanrahan JW, Bilodeau G, et al. Cystic fibrosis transmembrane conductance regulator function is suppressed in cigarette smokers. Am J Respir Crit Care Med 2006; 173: 1139-1144.

23 Rieber N, Hector A, Carevic M, et al. Current concepts of immune dysregulation in cystic fibrosis. Int J Biochem Cell Biol 2014; 52: 108-112.

24 Ordoñez CL, Henig NR, Mayer-Hamblett N, et al. Inflammatory and microbiologic markers in induced sputum after intravenous antibiotics in cystic fibrosis. Am J Respir Crit Care Med 2003; 168: 1471-1475.

25 Horsley AR, Davies JC, Gray RD, et al. Changes in physiological, functional and structural markers of cystic fibrosis lung disease with treatment of a pulmonary exacerbation. Thorax 2013; 68: 532-539.

26 Noah TL, Ivins SS, Abode KA, et al. Inhaled versus systemic antibiotics and airway inflammation in children with cystic fibrosis and Pseudomonas. Pediatr Pulmonol 2010; 45: 281-290.

27 Rosenfeld M, Gibson RL, McNamara S, et al. Early pulmonary infection, inflammation, and clinical outcomes in infants with cystic fibrosis. Pediatr Pulmonol 2001; 32: 356-366.

28 Ratjen F, Rietschel E, Griese M, et al. Fractional analysis of bronchoalveolar lavage fluid cytology in cystic fibrosis patients with normal lung function. Bronchoalveolar lavage for the evaluation of anti-inflammatory treatment (BEAT) study group. Eur Respir J 2000; 15: 141-145.

29 Gangell C, Gard S, Douglas T, et al. Inflammatory responses to individual microorganisms in the lungs of children with cystic fibrosis. Clin Infect Dis 2011; 53: 425-432. 